\title{
President's Column
}

\author{
Pat Smedley, MSc, BA Hons., RGN, RN, PGCE
}

A udit and its place in modern healthcare delivery. Clinical Audit: 'measuring the quality of care and services against agreed standards and making improvements where necessary' Health Quality Improvement Partnership.

Florence Nightingale was responsible! Well partly at any rate. Her famous wedge diagram (coxcomb) showing the different causes of death of British soldiers during the Crimean War was the result of statistical analysis of raw data - an early form of audit.

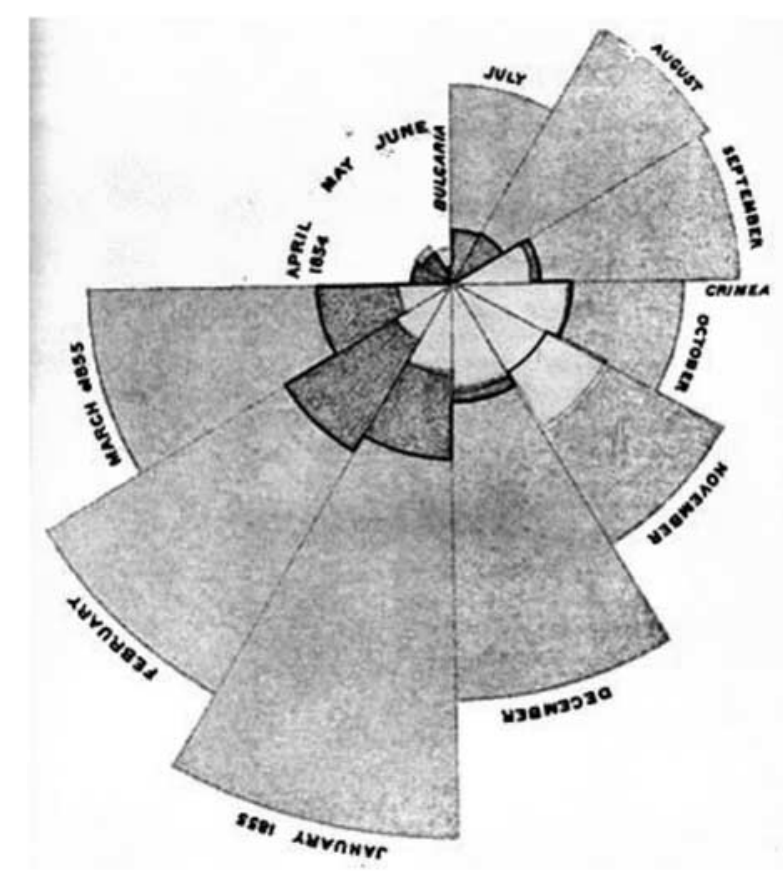

Nightingale's coxcomb (1857)

Nightingale went on to pioneer the use of survey/audit to gather data on health and living conditions both in military and civilian populations. By careful marshalling of the most accurate raw statistical data together with the enlistment of the finest expertise to help understand it, Nightingale was able to formulate solutions initially in Army health reform and from there to make possible health reform in all walks of life.

Fast forward now to 2012 where the standards and audit form the basis for all clinical activity within the clinical governance framework. National audit frameworks really came into existence at the end of the 1980s as the National Health Service (NHS) adopted the philosophy of the market place to improve standards. Healthy competition between the new style Trust hospitals would ensure that those who delivered the best services would gain the greatest rewards. The quality of service demanded written standards of care, and in the 1990s there was a rush to produce local standards - and then to audit those standards.

The Audit Commission was founded in 1983 as external auditors for local authorities in England and Wales - in 1990 its role was extended to the NHS. Its current remit includes more than 13,000 bodies, which between them spend $£ 100$ billion of public money annually.

The Hospital Episode Statistics (HES), a data warehouse containing details of all admissions into NHS hospitals in the United Kingdom, started collecting data from 1989. Before 1987, only $10 \%$ of admitted patients records were collected nationally. By 2006-2007, 13 million patient admission data records were stored out of which came 15 million episodes. The HES is the data source for a wide range of healthcare analysis system for the NHS 12 million news records are added each year. Data collected includes clinical information about diagnoses and operations - waiting list time, place of origin and so on. Personal information is not divulged but the information goes towards planning care: that is, how many women aged between 66 and 74 years living in Manchester underwent complex heart surgery in London hospitals during the period from April to September 2004? This provides invaluable information about average lengths of stay in hospitals and the time patients spent on waiting lists. Data on diagnoses, procedures and interventions, length of stay, waiting lists - all these data allow authorities to plan healthcare provision across the country. 
The year 2011 marked the 10th anniversary of the Dr Foster Hospital Guide. The mission for this leading independent agency was clear: transparency in outcomes, choice for the patient, accountability for the hospitals. The annual publication of statistics on hospital mortality rates and other hospital performance criteria (such as medical weekend cover) has helped to decrease the hospital death rates enormously. Open publication of these statistics has enabled patients to choose care more realistically.

The Healthcare Quality Improvement Partnership (HQIP) was established in 2008 to promote quality in healthcare, and to increase the impact that clinical audit has on healthcare quality in England and Wales. The HQIP is a huge umbrella organisation that believes in building both national and local level partnerships between clinical teams, managers and patients. Supporting local staff and fostering active dissemination of information and implementing quality improvements are key to its work. The establishment of the HQIP was part of the 2008 government initiative to make quality improvement with clinical audit at the heart - the centre for improving the NHS within the clinical governance framework.

Within the more specific field of perioperative care, the National Confidential Enquiry into Patient Outcome and Death (NCEPOD) was founded in 1988, it's first report being published in 1990. Its precursor was a confidential and anonymous pilot study of mortality associated with anaesthesia in order to improve clinical practice in anaesthesia. Since its inception NCEPOD has moved from reviewing the care of surgical patients and now covers all specialities. Latest audits around perioperative care published by NCEPOD are:

a. Perioperative care: Knowing the Risk (2011) in which deficiencies in the management of high-risk patients undergoing surgery were outlined. The highrisk group of 30 -day mortality was almost $7 \%$ and this encompassed three quarters of postoperative deaths. Recommendations included full investigation of high-risk patients in pre-assessment clinics with great assessment with correction of nutritional and fluid status both pre- and postoperatively.

b. Surgery in children: Are We There Yet? (2011). Key findings summarised an inadequate provision for children's surgery in NHS hospitals. Lack of data, provision of clinical networks for children, audit, operational policies, written information for children and parents about anaesthesia, poor theatre scheduling for children and lack of provision for suitable postoperative care environments for children all signified that there is some way to go in creating optimum conditions for caring for children in surgery.

Other national audits around peri-anaesthetic care have been lead by the Royal College of Anaesthetists who undertook four National Audit Projects (NAP) on major problems in anaesthesia. The third successful NAP (2006-2009) was about Central Neuraxial Blockade. The fourth NAP was an ambitious project being conducted jointly by the Royal College of Anaesthetists and the Difficult Airway Society in co-operation with the National Patient Safety Agency (NPSA). It commenced in September 2008 and ran for one year to determine the incidence of major complications of airway management in the United Kingdom. The report was published in mid 2010. The study demonstrated that obese patients are twice as likely to develop serious airway problems during a general anaesthetic than non-obese patients. The study also showed that the use of a capnograph, could significantly reduce deaths in the Intensive Care Unit where the problem was identified. The findings were published in March 2011. This was a new territory - the impact of airway complications is devastating and the opportunity to audit performance nationally and learn from a detailed analysis of such cases had never happened before.

The current and fifth NAP undertaken by the Royal College of Anaesthetists working with the Association of Anaesthetists of Great Britain and Ireland is about Accidental Awareness during Anaesthesia. The time period for audit is from June 2012 to May 2013. Further information on this topic may be available at www. nationalauditprojects.org.uk. This national audit will be administered by the newly formed Health Service Research Centre (HSRC), a department specifically to manage research and audit projects in anaesthesia.

Other national audits within perianaesthesia are via the Royal College of Nursing - which give information about how to audit into perioperative fasting (see www.rcn.org). The British Pain Society is currently doing a large national audit into pain - The National Pain Audit - funded by HQIP.

What of the future? There are a few certainties here:

- Clinical governance and audit is now embedded in every aspect of health care. Increasingly, sophisticated IT systems will ensure that hospitals routinely collect audit data on clinical and operational performance.

- Statistics related to hospital performance will be increasingly available to the general public to encourage choice and drive up quality as hospital trusts compete to offer the best services and attract more business. 
- Major professional organisations (such as the Royal College of Anaesthetists) will increasingly organise national audits to investigate problems in practice.

There is no doubt that high profile national audits, combining authority and topicality are hugely influential in instigating changes in clinical practice via a trickle down effect. National audit reports inform professional bodies and hospital authorities. Both of these feedback and impact on you - the nurse working at the coal face. By participating in local (and national) audit you help to create evidence that will in turn lead to improved performance and safer care for the patient.

British Anaesthetic \& Recovery Nurses Association (BARNA) vision statement: 'excellence in care through education, audit and research' clearly identifies how important audit is to this organisation. Our future development of audit within anaesthetic and recovery nursing will be:

- To inform members of national and local audits currently being undertaken via the website.
- To update Standards of Clinical Practice and make available on the website.

- To provide key information on how to go about clinical audit on the website.

- To conduct an annual audit into a key problem area in anaesthetic and recovery nursing in the United Kingdom - again audit forms and audit results will be posted on the website.

In 2010-2011, BARNA carried out a small-scale audit on staffing, the results of which are published in this journal. The results were interesting and confirmed the supposition that staffing remains a very difficult area for Post-Anaesthesia Care Unit managers. There is no doubt that a further more widespread audit needs to be carried out around staffing issues in the near future and position statements formulated. This is an ongoing work but fundamental to the aims of this association. It is no good just complaining about problems in clinical practice - opinions are just that - words without foundation. Audit is the tool that provides real data from which change can be enacted. 